CLINICAL PRACTICE

Clinical Images

\title{
The Art in Dermatitis
}

Shiao Yen Khoo, $\mathrm{MD}^{7}$, Carilyn N. Wieland, $M D^{2}$, and Mark L. Wieland, $M D^{7}$

'Department of Internal Medicine, Mayo Clinic, Rochester, MN, USA; ${ }^{2}$ Department of Dermatology, Mayo Clinic, Rochester, MN, USA.

KEY WORDS: contact dermatitis; allergic; henna.

J Gen Intern Med 27(8): 1079

DOI: $10.1007 / \mathrm{s} 11606-012-2018-4$

(C) Society of General Internal Medicine 2012

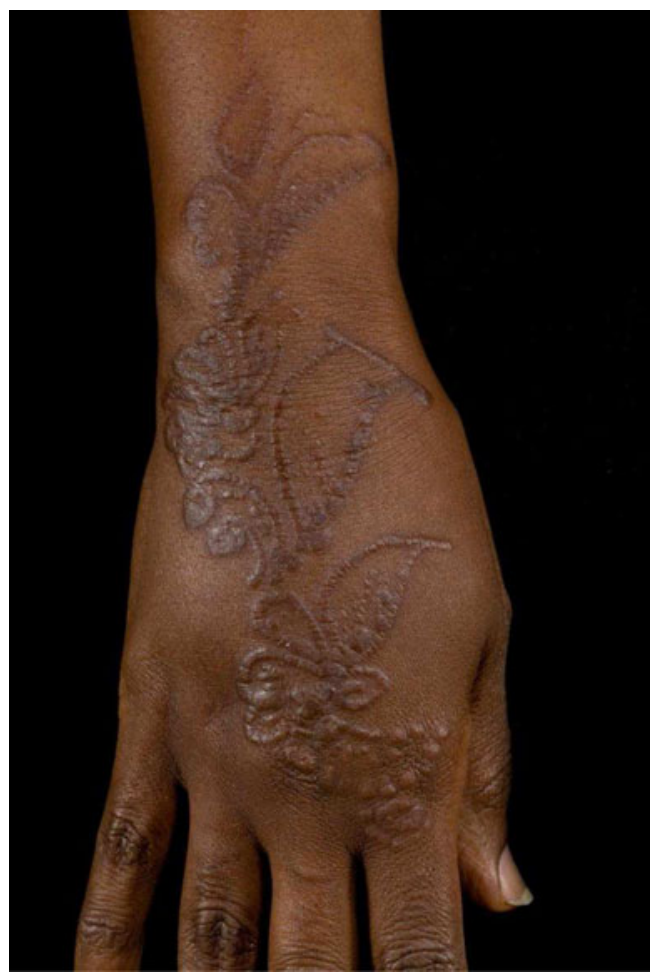

\begin{abstract}
32-year-old healthy Somali-American woman presented with one week of a pruritic papular rash over the dorsum of both hands. The rash appeared 7 days after her first henna application. There were no other new skin products identified. She was diagnosed with henna-induced allergic contact dermatitis and treated with a high potency topical corticosteroid and antihistamines with resolution of the lesions.

Henna is a plant native to Africa and southern Asia and is commonly used to dye skin and hair. Pure henna as a dyeing agent rarely causes skin sensitization.
\end{abstract}

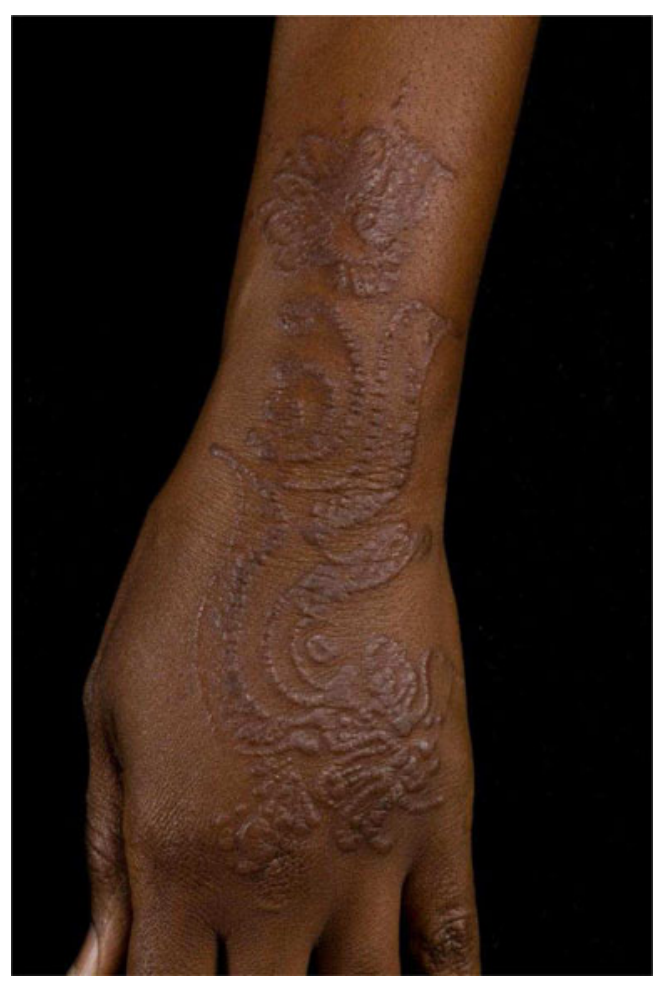

However, paraphenylenediamine (PPD) is often added to henna to speed drying and darken the dye. The addition of PPD to henna tattoo mixtures is increasingly being recognized as a cause of allergic contact dermatitis. Treatment consists of topical corticosteroids with or without antihistamines. In severe cases, systemic corticosteroids may be warranted. Post-inflammatory hyperpigmentation may occur following the resolution of the dermatitis.

Conflict of Interest: The authors declare that they do not have a conflict of interest.

Corresponding Author: Mark L. Wieland, MD; Department of Internal Medicine, Mayo Clinic, 200 First Street SW, Rochester, MN 55905, USA (e-mail: wieland.mark@mayo.edu). 